\title{
Study of a colliding laser-produced plasma by analysis of time and space-resolved image spectra.
}

\author{
H. Luna*, K.D. Kavanagh and J.T. Costello. \\ NCPST and School of Physical Science, Dublin City University, Glasnevin, Dublin 9, Republic of Ireland.
}

\begin{abstract}
The interaction of two counter-propagating laser-produced plasmas was studied using simultaneous imaging and spectroscopic techniques. Spectrally-filtered time-gated ICCD imaging was used to obtain information about the spatial dynamics and temporal evolution of the collision process. While, time-resolved imaging spectroscopy was used to determine the spatial and temporal distributions of electron temperature and density within the interaction region. We examine specifically the interaction of plasmas whose parameters match those typically used in pulsed laser deposition of thin films. These low temperature plasmas are highly collisional leading to the creation of a pronounced stagnation layer in the interaction region.
\end{abstract}

PACS numbers: 52.38.-r, 52.38.Mf, 52.70.Kz, 52.20.Hv

\section{INTRODUCTION}

When two expanding plasmas collide several interactions can arise. These interactions may be either of collisionless type, in which case collective plasma effects should occur or, in contrast they may be collision dominated. For example, considering two extreme cases where the ion-ion mean free path is either bigger or smaller than the typical dimension of the system, we can expect two different behaviours to occur during the interaction of the two plasma plumes. In the first case, the expanding plasma plumes should interpenetrate and therefore the heating processes should be mostly driven by binary collisions between the species. In the second case a quite different scenario is expected to occur once the two plasmas collide. In this case, the region of interpenetration should be relatively small and the two plumes will decelerate rapidly and stagnate. A stationary plasma should then be formed at the interface, if the translational (kinetic) energy of the plasma streams, is converted into thermal energy. This very localised heated region is frequently referred as the stagnation layer.

The study of the balance between stagnation layer formation versus interpenetration during the collision of two laser-produced plasmas, has been attracting attention over the past 30 years due to its broad range of possible applications to various fields in science such as: design of inertial confinement fusion hohlraums [1,2]; interaction of natural satellite atmospheres with solar winds and planet magnetosphere [3] (e.g., the production and heating of the Jovian magnetospheric plasma [4]); and experiments designed to address key aspects of the plasma physics occurring in supernova remnants [5].

In order to tailor the conditions under which the plasmas collide, different target configurations can be employed depending on the plasma collisionality required. The two most popular choices for target geometries are a single planar target [6] or an orthogonal-planar target [7]. Also, spectroscopic/imaging [7] and laser interferometric [5, 8] techniques have been employed in the study of colliding plasmas.

Previously, Harilal and co-workers [7] have studied the temporal and spatial expansion dynamics of colliding laser produced magnesium plasma at very early times using a gated XUV pinhole imaging technique. They subsequently, extended their work to spectroscopic studies in the XUV range [9]. Later Atwee and Kunze [10] applied the same technique to study the collision of boron nitride laser-produced plasmas. In all works mentioned above the focus was on intermediate temperature plasmas with electron temperatures $\left(\mathrm{T}_{e}\right)$ in the range of tens to some hundreds of electron volts.

In the present work we extend the study of laser-produced colliding plasmas to low temperature plumes $\left(\mathrm{T}_{e}<5\right.$ $\mathrm{eV}$ ). These low temperature plasmas are similar to those used in pulsed laser deposition of thin films. In the following section (sec. II) we will describe the imaging technique [11] used in this work to obtain fully resolved spectroscopic images (spatial and temporal). From these images we were able to track the collision dynamics, as well as to extract plasma spectroscopic information such as line intensities and broadening parameters. In section III we will present images of the colliding plasma dynamics followed by interaction and stagnation layer formation. Plasma parameters such as electron temperature and density spatial distributions, obtained from spectroscopic analysis of the stagnation layer, will then be presented and discussed in sectionIV.

* Author to whom any correspondence should be addressed: hdl@physics.dcu.ie 


\section{EXPERIMENTAL APPARATUS}

The experimental apparatus is shown schematically in figure 1 and is similar to that already described in reference [11]. Hence only the salient details will be given here. Briefly, the third harmonic $(\lambda=355 \mathrm{~nm})$ of a Q-switched Nd:YAG laser (Surelite ${ }^{\mathrm{TM}}$, pulse duration of $6 \mathrm{nsec}$ and $10 \mathrm{~Hz}$ repetition rate) was split into two beams by a movable glass wedge with a 2 degree acute angle. The two beams were subsequently focused onto a V-shaped cut in the surface of a calcium slab (as shown in the inset of figure 1), located inside a vacuum chamber $\left(5 \times 10^{-5}\right.$ mbar $)$. Two plasmas were generated and expanded orthogonally towards each other. This geometry resulted in a distance between the two foci at the target surface of approximately $1.9 \mathrm{~mm}$. The target holder was mounted on an $X Y Z$ stage in order to allow us to move to a new surface after exposure. The laser irradiance was kept constant at an average of $3 \times 10^{10} \mathrm{~W} / \mathrm{cm}^{2}$.

The optical system used to focus the plasma onto the entrance slit of the spectrometer included a Dove prism located between two achromatic lenses, $l_{2}$ and $l_{3}$ with focal lengths of $250 \mathrm{~mm}$ each. The Dove prism was used to rotate the image of the expanding plasma plume by 90 degrees, projecting the direction of the plasma expansion (longitudinal) onto the entrance slit of a $0.5 \mathrm{~m}$ focal length Czerny-Turner spectrometer (Chromex 500is/sm). The spectrometer was equipped with a grating of groove density of $1200 \mathrm{~mm}^{-1}$ and blaze wavelength of $400 \mathrm{~nm}$. The optimum resolution was obtained at a slit width of $60 \mu \mathrm{m}$ since no improvement in resolution was obtained at narrower slit widths while the resolution was degraded at larger slit widths. The magnification of the optical system was 1 : 1 . Spectral image readout was provided by an (Andor $\left.{ }^{\mathrm{TM}} \mathrm{ICCD}\right)$. The ICCD consisted of a $18 \mathrm{~mm}$ diameter Micro-Channel Plate (MCP) image intensifier coupled to a $1024 \times 1024$ pixels Charge-Coupled Device by a high quality relay lens system. Since the spectrometer is stigmatic, the overall ICCD/spectrometer system provided spectra of the plasma plume which were spatially resolved along the plume expansion direction.

A semi-silvered mirror, placed at 45 degrees between the vacuum chamber and $l_{2}$, permitted a simultaneous 2 -D (plane) measurement of plasma plume spatial expansion with the aid of a second ICCD equipped with a $512 \times 512$ pixels array. These 2-D images were also spectrally resolved using a set of narrow band pass interference filters. This permitted us to track the spatial distribution of emission from a specific transition. The magnification of the optical system was found to be $1: 1.3$.

The ICCD cameras used in the experiment could be operated with a gate width as short as 3 ns. By varying the delay between plasma initiation and the opening of the ICCD's gate, we were able to track the temporal evolution of the plasma. Two Stanford DG535 ${ }^{\mathrm{TM}}$ delay generators were used to achieve this synchronization. A gate width of 5 nsec was used to record all images and spectra presented here.

\section{EXPANSION DYNAMICS}

Images of plasma expansion (2-D plane) followed by subsequent interaction and stagnation were obtained for different times after initial plasma plumes formation. The $3 \mathrm{p}^{6} 4 s\left({ }^{2} S_{1 / 2}\right) \rightarrow 3 \mathrm{p}^{6} 4 p\left({ }^{2} P_{3 / 2}\right)$ Ca II optical transition, with $\lambda=393.36 \mathrm{~nm}$, was used to track the spatial/temporal evolution of the singly charged component of the two plumes. This was accomplished by using a band pass filter, $10 \mathrm{~nm}$ wide, centered at $390 \mathrm{~nm}$ in front of the $512 \times 512$ pixels ICCD. In figure 2 we show the spatial and temporal evolution at; (a) $100 \mathrm{~ns}$, (b) $200 \mathrm{~ns}$, (c) 500 ns, and (d) 700 ns after plasma initiation. The ICCD gain was kept constant at $50 \%$ of maximum throughout the measurements. By monitoring the single plume displacement, taking as a reference point the maximum plume intensity, a longitudinal velocity of $11 \pm 1 \times 10^{5} \mathrm{~cm} / \mathrm{s}$ was obtained.

We note that, in contrast to XUV emission from colliding plasmas [7] which have initial temperatures of some tens of $\mathrm{eV}$, the duration of emission from the stagnation layer formed at the interface between these rather low temperature plasma is much longer. In the former case, strong (XUV) emission persists for only some tens of nanoseconds while for the latter case we can record images and spectra for time delays extending out past one microsecond. This is not a particularly surprising observation since XUV excitation will require plasma temperatures which are a reasonable fraction of the photon energy. As a result the stagnation layer plasma cools rather rapidly and the high photon energy portion of the emission concomitantly falls away in a short period of time. In contrast, in our experiments, the plasma plumes give rise to a layer with a much lower temperature and hence the emission lies predominantly in the visible and near-IR band while the temperature drops more slowly in time. Therefore we can observe and track the stagnation layer dynamics for much longer time periods.

In figure 3 we show the total emission from the Ca II component of the stagnation layer, obtained by spatially integrating over each 2D image (similar to figure 2) for a range of time delays from 150ns to $700 \mathrm{~ns}$. It is clear from this figure that full width half maximum (FWHM) of the emission has a value of almost 300 ns but can be even longer depending on initial plasma parameters. The FWHM of the stagnation layer at 500 ns time delay, near the peak intensity point, is $0.5 \mathrm{~mm}$ (Figure 2(c)). We found that this value fluctuates only slightly for time delays from 200 to $600 \mathrm{~ns}$ indicating that the layer is maintained in a relatively tight compressed state during this time interval. 


\section{SPECTROSCOPY}

Using the experimental procedure described in section II several isolated Ca emission lines were measured in the visible range, namely the Ca II doublet $3 \mathrm{p}^{6} 4 \mathrm{~s}\left({ }^{2} \mathrm{~S}_{1 / 2}\right) \rightarrow 3 \mathrm{p}^{6} 4 \mathrm{p}\left({ }^{2} \mathrm{P}_{3 / 2,1 / 2}\right)$ and the $3 \mathrm{p}^{6} 4 \mathrm{p}\left({ }^{2} \mathrm{P}_{1 / 2,3 / 2}\right) \rightarrow 3 \mathrm{p}^{6} 5 \mathrm{~s}\left({ }^{2} \mathrm{~S}_{1 / 2}\right)$ transitions corresponding to the wavelengths of 393.36, 396.84, 370.60 and $373.69 \mathrm{~nm}$, respectively. We have also measured the neutral emission singlet $3 \mathrm{p}^{6} 4 \mathrm{~s}^{2}\left(\mathrm{~S}_{0}\right) \rightarrow 3 p^{6} 4 \mathrm{~s} 4 \mathrm{p}\left({ }^{1} \mathrm{P}_{1}\right)$, which has a wavelength of $422.67 \mathrm{~nm}$. In figure 4 we show a typical spectroscopic image for the 370.60 and $373.69 \mathrm{~nm}$ doublet at $420 \mathrm{~ns}$ after plasma initiation, corresponding to a delay of approximately $270 \mathrm{~ns}$ after stagnation layer formation.

Particular care was taken in the alignment of the stagnation layer plasma, optics and spectrometer in order to avoid contamination from the emission of the expanding single plasma plumes. A plot of the spatial distribution of the integrated intensity of each of the five measured spectral lines as a function of distance along the stagnation layer is shown in figure 5. The total intensity for each line is obtained using the procedure described in [11]. Briefly, we overlay the area of interest in the spectral image with a grid containing 32 boxes covering a longitudinal distance of approximately $6 \mathrm{~mm}$. One such box is shown in figure 4 for illustration. For each time delay and each individual spectral line we integrate over the binned portion of the spectrum to obtain a measure of the space resolved intensity. Data shown in figure 5 were obtained from spectra taken at a time delay of $300 \mathrm{~ns}$ where the integrated intensity is shown on a log scale. It is clear that the peak intensity occurs some $2 \mathrm{~mm}$ from the edge of the layer closest to the target for all lines. It is also clear that Ca II 393.36/396.84 nm doublet yields the strongest emission followed by the Ca II $373.69 \mathrm{~nm}$, Ca I $422.67 \mathrm{~nm}$ and the Ca II $370.60 \mathrm{~nm}$ transitions. However, our main interest in tracking the evolution of these lines in space and time was to determine the spatio-temporal distribution of the electron density and temperature in the stagnation layer.

\section{A. Density.}

The electron density was calculated via an analysis of the profile of the Ca II 373.69 line. A Voigt analysis is used to fit a profile to the transition that takes into account the various processes that contribute to the line broadening. The Stark contribution to the broadening is described by the Lorentzian component and all other contributions, such as Doppler effect (order of $0.005 \mathrm{~nm}$ for a plasma temperature of $1 \mathrm{eV}$ ) and the instrument function of $\delta=0.11 \mathrm{~nm}$ (measured independently) were accounted for the Gaussian component of the Voigt profile [12, 13]. Furthermore, having obtained the pure Stark broadened component, the electron density can be computed from the line widths by solving the equations below:

$$
\begin{aligned}
\Delta \lambda_{w i d t h} & =2 W\left(N e / 10^{16}\right)+3.5 A\left(N e / 10^{16}\right)^{1 / 4} \\
& \times\left[1-1.2 N_{D}^{-1 / 3}\right] W\left(N e / 10^{16}\right)
\end{aligned}
$$

where the electron impact parameter $W$, and the ionic-impact parameter $A$ are taken from tabulated values [12] and $N_{D}$ is the number of particles in the Debye sphere.

The first term in equation 1 describes the broadening caused by electron-emitter collisions. The second term, or quasi-static component describes the broadening due to ion-emitter interactions. Stark broadening in lowly ionised species is dominated by electron collisions, thereby allowing us to use only the first term in the equation 1 . The FHWM $\left(\Delta \lambda_{\text {width }}\right)$ will then scale linearly with the electron density $N_{e}$.

The spatial distribution of the electron density for times in the vicinity of the peak brightness epoch, $300 \mathrm{~ns}$ to 500 $\mathrm{ns}$, are shown in figure 6 . It should be noted that we first see light emission from the stagnation layer as it begins to form at about $150 \mathrm{~ns}$ after plasma initiation and so one should subtract this number from these time delays to get times following stagnation layer birth. Not unexpectedly the peak in the electron density coincides approximately with the position of the emission intensity peak (see figure 5 again) since the radiative processes in this thermal plasma will be largely electron mediated. The electron density distribution remains reasonably constant along the stagnation layer with the peak value dropping away slowly during our observation times.

\section{B. Temperature.}

Under the assumption of LTE, the electron temperature in the plasma can be obtained from the spectroscopic measurements by: (1) relative line to continuum intensity, (2) relative intensity of lines from the same ionization stage, and (3) relative line intensity of subsequent ionization stages. 
The relative line to continuum method, although very useful in the characterization of early stages of a single plasma plume expansion, could not be used in the present work due to the absence of continuum emission from the stagnation layer. The relative intensity of lines from the same ionization stage obtained from the ratio of the Ca II $3 \mathrm{p}^{6} 4 \mathrm{~s}\left({ }^{2} \mathrm{~S}_{1 / 2}\right) \rightarrow 3 \mathrm{p}^{6} 4 \mathrm{p}\left({ }^{2} \mathrm{P}_{3 / 2,1 / 2}\right)$ to $3 \mathrm{p}^{6} 4 \mathrm{p}\left({ }^{2} \mathrm{P}_{1 / 2,3 / 2}\right) \rightarrow 3 \mathrm{p}^{6} 5 \mathrm{~s}\left({ }^{2} \mathrm{~S}_{1 / 2}\right)$ doublets, was avoided due to the presence of significant opacity in the $4 \mathrm{~s}\left({ }^{2} \mathrm{~S}_{1 / 2}\right) \rightarrow 4 \mathrm{p}\left({ }^{2} \mathrm{P}_{3 / 2,1 / 2}\right)$ transitions for our plasma conditions.

However, if one observes two isolated lines of subsequent ionization stages, $\lambda$ and $\lambda^{\prime}$, emitted from the same atomic or ionic species, and if the energy level population is distributed according to the Boltzmann law, the line intensity ratio is given by [12]:

$$
\frac{I^{\prime}}{I}=\frac{f^{\prime} g^{\prime} \lambda^{3}}{f g \lambda^{\prime 3}}\left(4 \pi^{3 / 2} a_{0}^{3} N e\right)^{-1}\left(\frac{k T}{E_{H}}\right)^{3 / 2} \exp \left(\frac{E-E^{\prime}-E_{\infty}}{k T}\right)
$$

where $I, \lambda, g$ and $f$ are: total intensity (integrated over the profile), wavelength, statistical weight of the lower state of the line, and absorption oscillator strength, respectively, of the line related to the lower charge state transition and $E$ its excitation energy and $a_{0}$ denotes the Bohr radius. The subsequent ionization stage is denoted by primed quantities, and $E_{\infty}$ is the ionization energy of the lower ionization stage. The relative intensities have to be corrected according to the wavelength dependence of the spectral sensitivity of the ICCD and of the grating transmission efficiency. We have employed the same procedure described in our previous work [11] where an intensity calibrated light source was used to measure the relative total spectral efficiency of our system (ICCD plus spectrometer).

Temperatures were obtained by solving the equation 2 using a graphical method, as illustrated in figure 7 . The line intensity ratios were computed from the ratio of Ca II $373.69 \mathrm{~nm}$ and Ca I $422.7 \mathrm{~nm}$ transitions. This graphical method permits temperature determinations with accuracies ranging from 5 to $15 \%$, for temperatures between 0.55 and $2 \mathrm{eV}[12]$.

The spatial temperature distribution along the stagnation layer is shown in figure 8 for three times near the maximum brightness, namely 300, 420 and $500 \mathrm{~ns}$ after plasma initiation or equivalently 150,270 and 350 ns after the stagnation layer formation. Our results show a nearly iso-thermal distribution of approximately $1.4 \mathrm{eV}$ along the major axis of the longitudinal stagnation layer.

As pointed out above, the $3 \mathrm{p}^{6} 4 \mathrm{~s}\left({ }^{2} \mathrm{~S}_{1 / 2}\right) \rightarrow 3 \mathrm{p}^{6} 4 \mathrm{p}\left({ }^{2} \mathrm{P}_{3 / 2,1 / 2}\right)$ doublet was avoided in the spectroscopic analysis because of opacity. For a colliding plasma, a self reversed profile was found to be present into a region localised near the maximum intensity/density. However, temperatures obtained from regions where the self-reversal profile seemed not to be present, were still systematically smaller when calculated using the doublet. This can be regarded as an indication that part of the emission from the 393.36/396.84 nm doublet is still being reabsorbed by the cooler part of the plasma and that the dip in each profile is washed out by instrumental broadening. Great care has to be taken in analysing such profiles. We are currently developing a model to assist in this process based on the work of Sakka et al $[15]$.

Finally we note that, in contrast to stagnation layers formed by colliding relatively high temperature plasma [9, 10], we do not observe any significant continuum emission from the stagnation layer even at early times after its formation. Any continuum emission will result from either free-free (bremsstrahlung) or free-bound (recombination) processes and its absence here is an indication that we observe almost pure stagnation with little or no interpenetration. The result can be understood by looking at the form of the collisionality parameter used to characterise the situation. For a pair of colliding plasma the collisionality parameter $(\zeta)[16,17]$ can be cast as the ratio of the characteristic geometrical scale length $(\mathrm{D})$ to the ion-ion mean free $\operatorname{path}\left(\lambda_{i i}\right)$. So for plasma with high ion density $(\mathrm{n}(x))$ and a gentle spatial rolloff (large plasma scale length - $\mathrm{dn} / \mathrm{d} x$ ) formed in close proximity to each other (small D), one can expect strong deceleration at the plasma-plasma collision front and the formation of a tight, well defined stagnation layer. Since the ion-ion mean free path $\left(\lambda_{i i}\right)$ depends on the ratio $\left(\mathrm{T}^{2} / \mathrm{n}_{i}\right)$, low temperature plasmas will possess quite low values of $\lambda_{i i}$ and concomitantly high values of $\zeta$ and hence almost no interpenetration can be expected. Rough estimates for our case $\left(\mathrm{T}_{e} \sim 1.4 \mathrm{eV}, \mathrm{n} \sim 10^{18} \mathrm{~cm}^{-3}\right)$ give $\zeta$ values of $\sim 10^{4}$ while for Harilal et al. [9] the temperature can be expected to be $>30 \mathrm{eV}$ (strong emission from $\mathrm{Mg}$ ions with charge $>5^{+}$), and hence the collisionality parameter is likely to be $<10$ and therefore interpenetration is expected, as observed in their work.

\section{CONCLUSION}

We have observed the formation of a stagnation layer at interface between two low temperature counter-propagating laser plasma plumes. We have studied the time and space evolution of the stagnation layer using optical imaging and spectroscopy. The stagnation layer emits strongly in the visible region of the spectrum for some hundreds of nanoseconds and the emission distribution along the long axis of the cylindrical shaped plume follows the electron 
density distribution quite closely. The temperature distribution at times near 300 ns after initial plasma ignition (or $150 \mathrm{~ns}$ after stagnation layer formation) is nearly iso-thermal and typically $>1 \mathrm{eV}$. For our case, and in contrast to most other work in this field, our conditions favour the formation of a strong, well defined stagnation region with almost no interpenetration.

\section{A. Acknowledgments}

This work was supported by the Irish Government National Development Plan under the Frontiers (Basic Research Grants Scheme) of Science Foundation Ireland. KDK acknowledges support from the Embark Scholarship Scheme of the Irish Research Council for Science Engineering and Technology. HL acknowledges support from the EU FP6 Marie Curie program and HEA North-South programme.

References.

[1] Kunze H-J, Koshelev K N, Steden C, Uskov D and Wieschebrink H T 1994 Phys. Lett. A193 183

[2] Clark R W, Davis J, Velikovich A L and Whitney K G 1997 Phys. Plasmas 43718

[3] Galeev A A, Cravens T E and Gombosi T J 1985 Astrophys. J. 289807

[4] Luna H, Michael M, Shah M B, Johnson R E, Latimer C J, and McConkey J W 2005 J. Geophys. Res. 108(E4) 5033

[5] Woosley N C, Courtois C and Dendy R O 2004 Plasma Phys. Control. Fusion 46 B397

[6] Henc-Bartolic V, Andreic Z, Gracin D, Aschke L, Ruhl F and Kunze H-J 1998 Phys. Scr. T 75297.

[7] Harilal S S, Bindhu C V, and Kunze H-J 2001 J. Appl. Phys. 894737.

[8] Wan A S, Barbee Jr. T W, Cauble R, Celliers P, Da Silva L B, Moreno J C, Rambo P W, Stone G F, trebes J E and Weber F 1997 Phys. Rev.E 556293.

[9] Harilal S S, Bindhu C V, Shevelko V P and Kunze H-J 2001 J. Phys. B: At. Mol. Opt. Phys. 343717.

[10] Atwee T and Kunze H-J 2002 J. Phys. D: Appl. Phys. 35524.

[11] Doria D, Kavanagh K D, Costello J C and Luna H 2006 Meas. Sci. Technol. 17670.

[12] H. R. Griem 1964 Plasma Spectroscopy (McGraw-Hill, New York).

[13] H. R. Griem 1974 Spectral Line Broadening by Plasmas (Academic Press, New York).

[14] NIST database, http://physics.nist.gov/PhysRefData/.

[15] Sakka T, Nakajima T and Ogata Y H 2002 J. Appl. Phys. 922296.

[16] Courtois C, Grundy R A D, Ash A D, Chambers D M, Dendy R O, McClements K G and Woosley N C, 2005 Astrophysics and Space Science 298: 9398.

[17] Rambo P W, and Denavit J, 1994 Physics of Plasmas 12: 4050-4060. 


\section{Figure Caption}

FIG. 1: Experimental set-up for the colliding plasma experiment. Ca target, lenses $\left(l_{1}, l_{2}\right.$ and $\left.l_{3}\right)$, wedge prism (WP), Dove prism, silver mirror (SM), filter wheel (FW), spectrometer and ICCD cameras.

FIG. 2: Temporal and 2-D spatial plasma evolution recorded at a time delay of: (a) $100 \mathrm{ns,} \mathrm{(b)} 200 \mathrm{ns,} \mathrm{(c)} 500 \mathrm{~ns}$ and (d)700 ns from plasma initiation. A narrow band pass filter centered at $390 \mathrm{~nm}$ was used to track the Ca II $3 \mathrm{p}^{6} 4 \mathrm{~s}\left({ }^{2} \mathrm{~S}_{1 / 2}\right) \rightarrow$ $3 \mathrm{p}^{6} 4 \mathrm{p}\left({ }^{2} \mathrm{P}_{3 / 2,1 / 2}\right)$ transition.

FIG. 3: Temporal evolution of the integrated emission intensity from the stagnation layer obtained from the 2-D images of figure 2 . 
FIG. 4: Typical raw spectroscopic image (wavelength on $\mathrm{x}$-axis and longitudinal distance on $\mathrm{y}$-axis) of the staganation region in the vicinity of the CaII doublet $370.60 \mathrm{~nm}$ and $373.69 \mathrm{~nm}$. The spectrum was recorded at a time delay of $420 \mathrm{~ns}$ from plasma initiation.

FIG. 5: Longitudinal intensity distribution for $300 \mathrm{~ns}$ from plasma initiation, for Ca transitions measured in this work.

FIG. 6: Longitudinal density distribution for 300, 420 and 500 ns from plasma initiation.

FIG. 7: Intensity ratio of Ca II $373.69 \mathrm{~nm}$ and Ca I $422.7 \mathrm{~nm}$ transitions as a function of the temperature (Eq.2) for various electron densities.

FIG. 8: Longitudinal temperature distribution for 300, 420 and 500 ns from plasma initiation. 


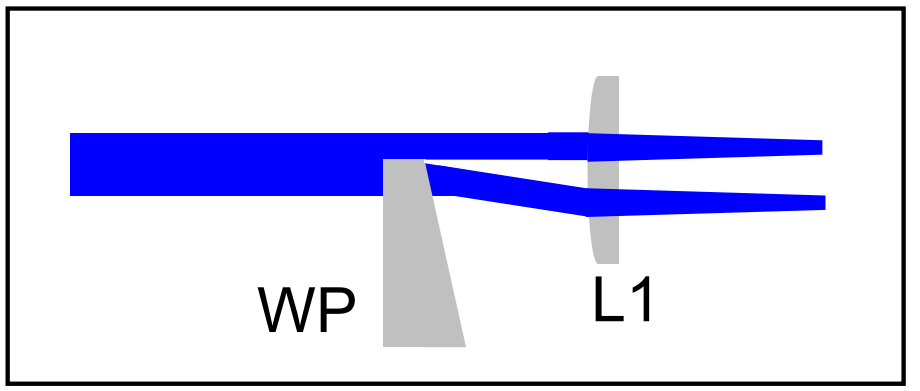

Ca target

Spectrometer

\section{ICCD-Camera}

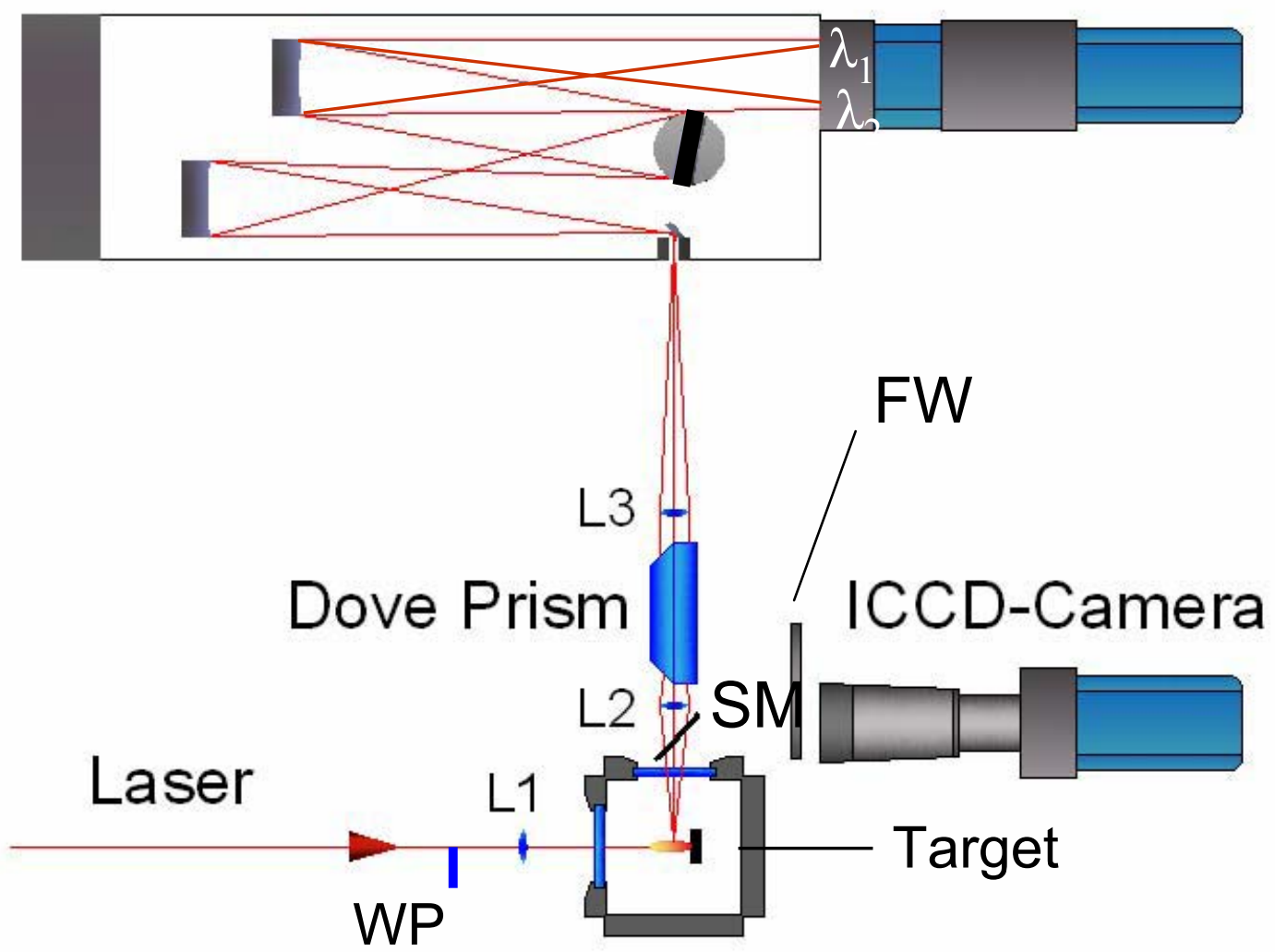




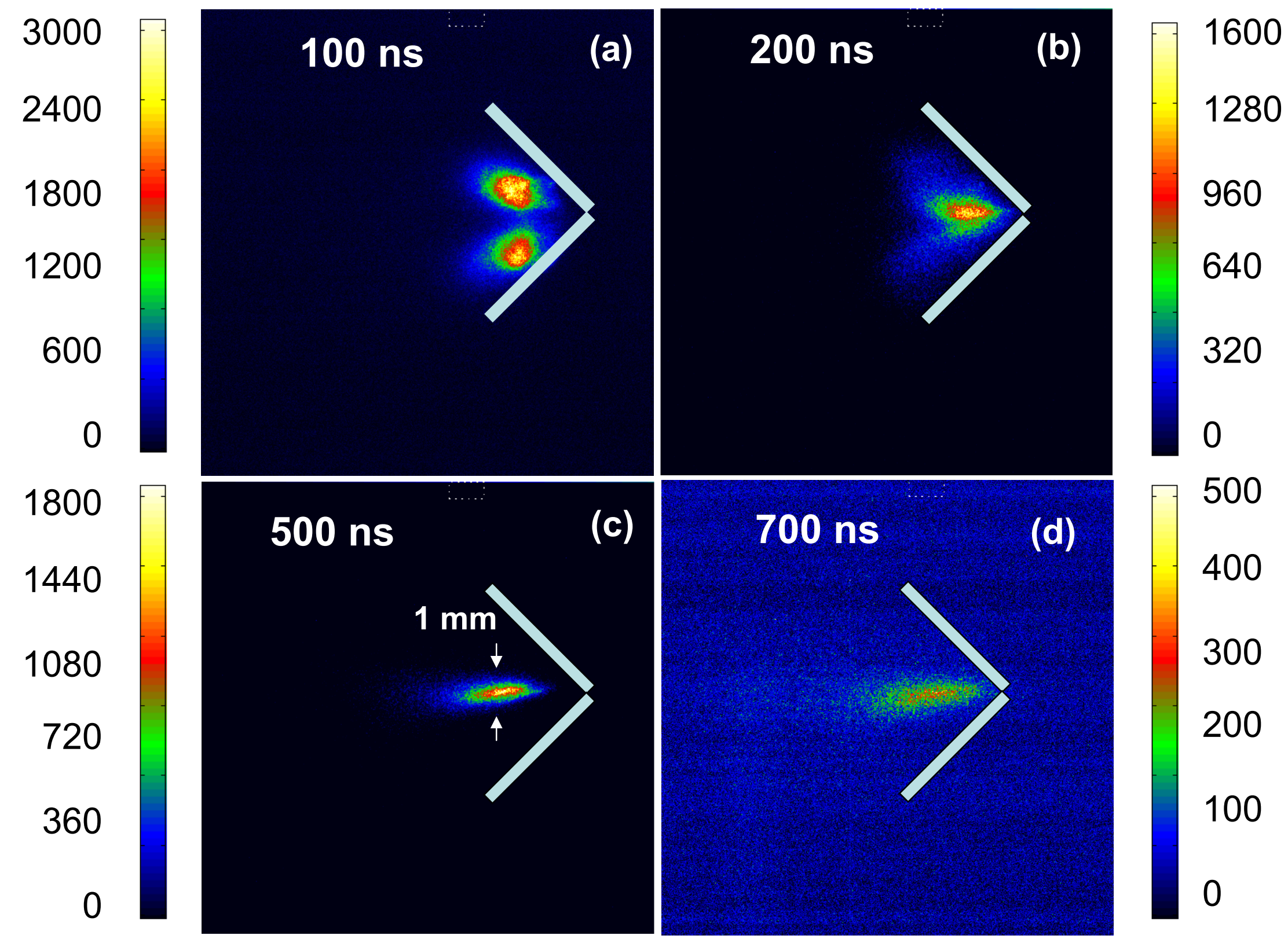




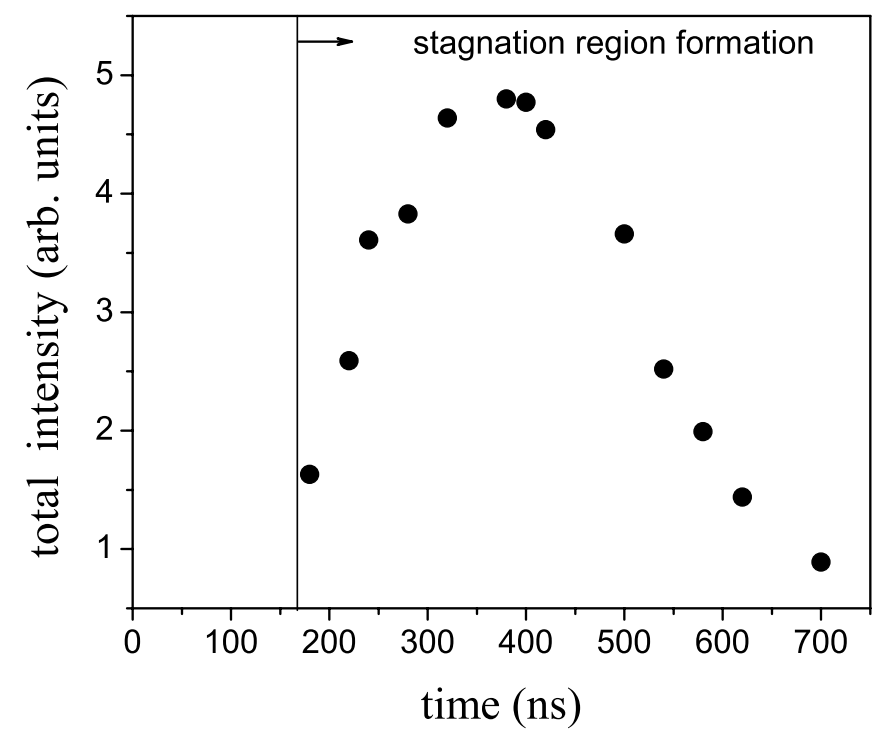




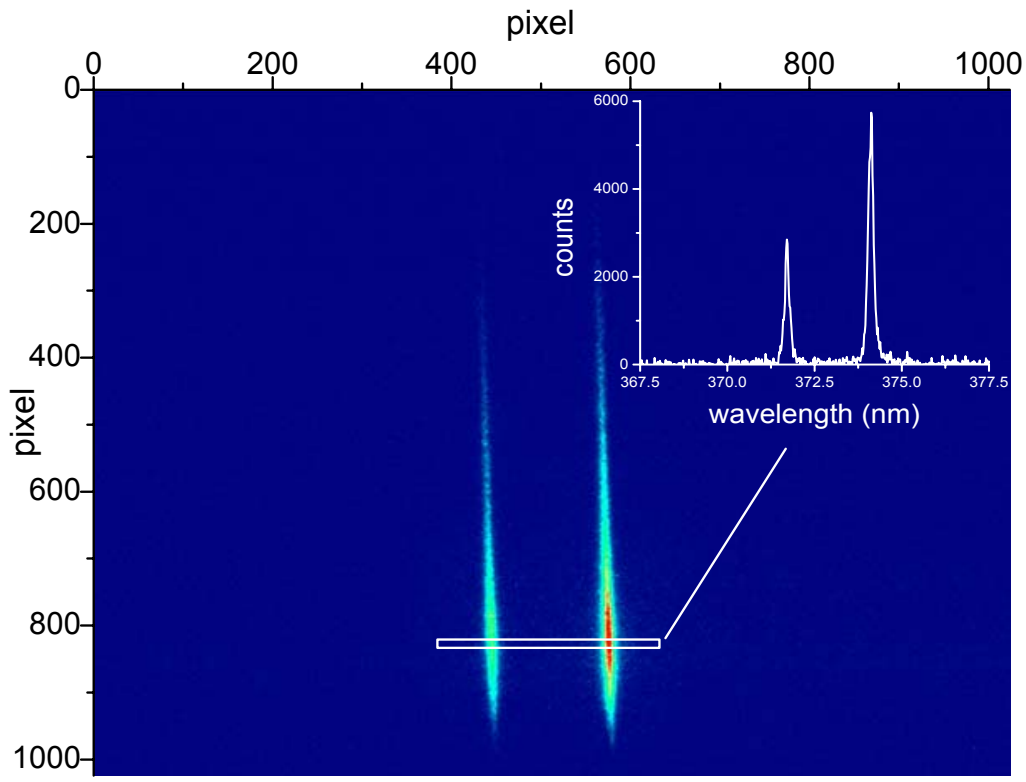




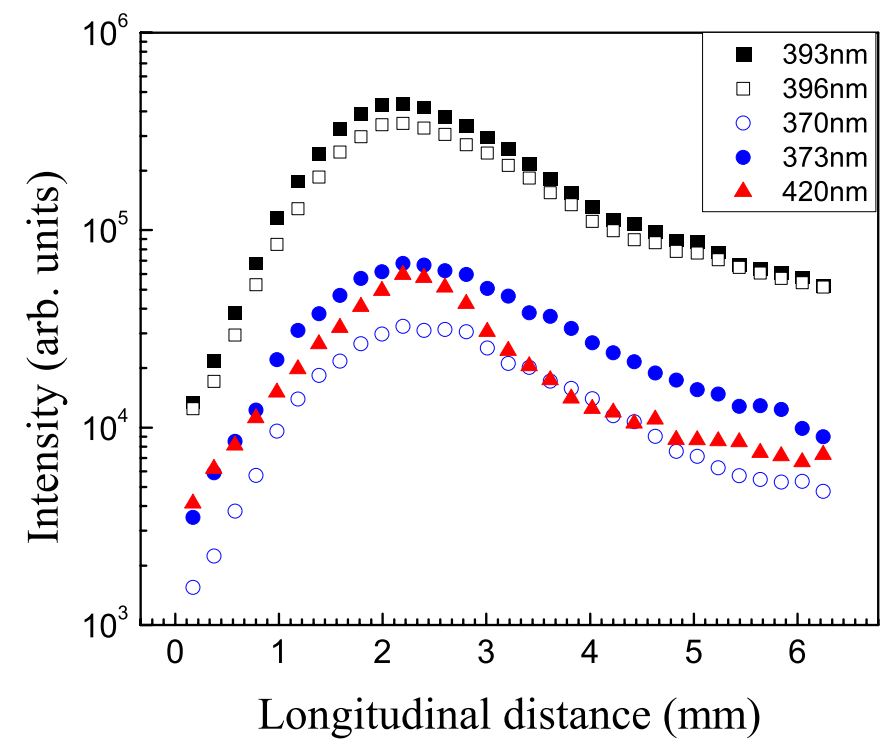



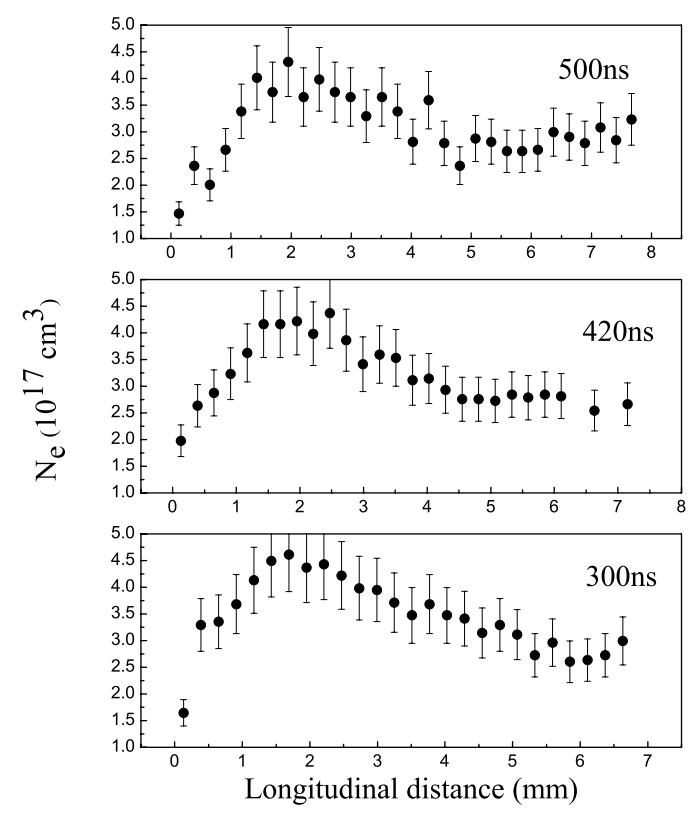


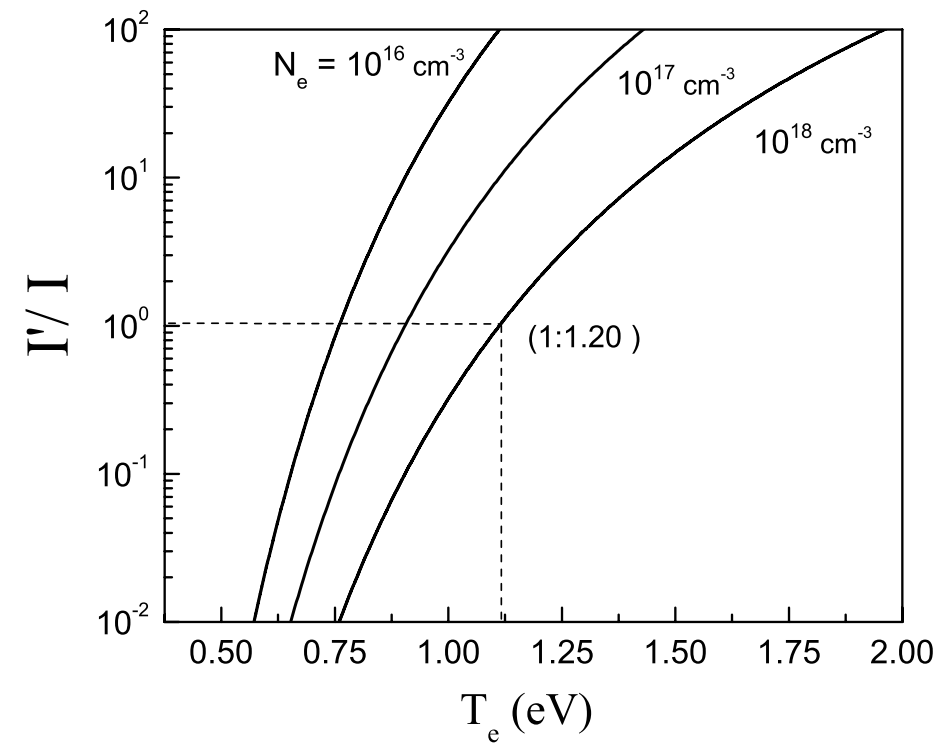




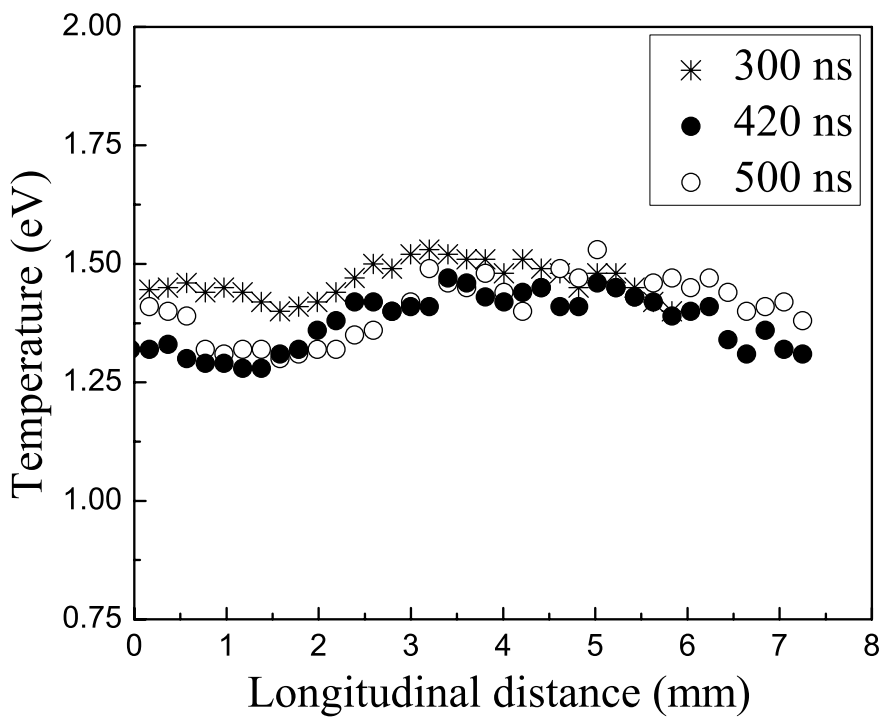

